\title{
Systeme der Künstlichen Intelligenz
}

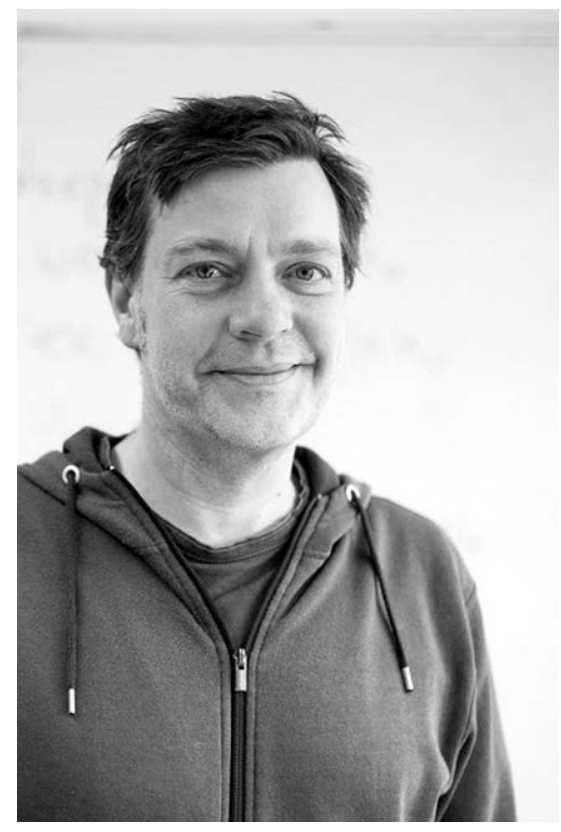

Systeme der Künstlichen Intelligenz finden in einer steigenden Anzahl von kritischen Lebensbereichen Anwendung, wie zum Beispiel bei der Diagnose von Krankheiten und der Personalisierung von Behandlungsmethoden, bei der Vorauswahl von Bewerber*innen für Arbeitsplätze, bei der Erstellung und Präsentation personalisierter Anzeigen, bei der Erkennung von Falschmeldungen und beim Filtern von Nachrichten. Systeme, die derartig stark in unsere Gesellschaft eingreifen, müssen hohen Anforderungen genügen: Sie sollten robust gegenüber Manipulationen sein, die Privatsphäre respektieren, Personen nicht diskriminieren und nachvollziehbar arbeiten.

Künstliche Intelligenz hat großes Potential, uns in vielen Lebensbereichen zu unterstützen. Gleichzeitig bringt diese neue Technologie, wie häufig, aber auch Gefahren mit sich. Um Klarheit über die Chancen und Risiken der Künstlichen Intelligenz zu erhalten, bedarf es zum einen einer klaren Formulierung der Anforderungen an diese Systeme, z.B. Sicherheit, Datenschutz und Diskriminierungsfreiheit, zum anderen müssen die Grundlagen dafür geschaffen werden, diese Anforderungen sowohl umsetzen als auch nachweisen zu können. Darüber hinaus ist natürlich auch die Gesetzgebung aufgefordert, die rechtlichen Rahmenbedingungen für die Umsetzung der notwendigen Anforderungen zu schaffen. Und nicht zuletzt muss auch die Zivilgesellschaft in diese Prozesse eingebunden werden, um Vertrauen in diese alles durchdringende Technologie zu fördern.

All diese Themen werden im Schwerpunkt der vorliegenden Ausgabe beleuchtet. So geht der Beitrag von Franziska Boenisch auf das Thema Privatsphäre in Machine Learning Modellen ein, Karla Markert und Konstantin Böttinger erläutern Angriffe auf KI-Systeme und diskutieren mögliche Gegenmaßnahmen und Marina Höhne erklärt das Thema nachvollziehbare Künstliche Intelligenz. Elisa Brummel und Marc Fliehe erläutern in ihrem Beitrag, welche Schwierigkeiten derzeit bei der Bewertung von KI-Systemen bestehen und warum eine Bewertung dennoch wichtig für das Vertrauen in diese Systeme ist.

Ein wichtiges Anliegen der Autoren Christian Kastrop und Dominic Ponattu ist es, die Zivilgesellschaft einzubinden. Sie fordern ein breites, zivilgesellschaftliches Ökosystem für eine verbraucherzentrierte Künstliche Intelligenz. Der Beitrag von Martin Schallbruch gibt einen Überblick, wie eine Regulierung künstlicher Intelligenz erfolgen soll und welche Aktivitäten hier von den Gesetzgebern verfolgt wird. Abgerundet wird der Schwerpunkt von Christian Thiel, Christoph Thiel, Arno Fiedler, die an Hand eines konkreten Anwendungsfalls, der automatisierten Personenidentifizierung, die Chancen und Risiken der Künstlichen Intelligenz diskutieren.

Zusätzlich zum Schwerpunkt enthält dieses Heft zwei Aufsätze. 'Rechtsschutz gegen Persönlichkeitsrechtsverletzungen im Netz - Prozedurale Gewährleistungen' von Louisa Specht-Riemenschneider und Lea Lorbach sowie,'Das Verbandssanktionengesetz-E und betriebliche Datenschutzbeauftragt' von Peter Gola.

Ich wünsche Ihnen viel Spaß bei der Lektüre.

\section{Marian Margraf}

